\title{
COMPARISON OF STOCHASTIC VOLATILITY MODELS: EMPIRICAL STUDY ON KOSPI 200 INDEX OPTIONS
}

\author{
Kyoung-Sook Moon $\dagger$, Jung-Yon Seon, In-Suk Weeł, And Choongseok Yoon
}

\begin{abstract}
We examine a unified approach of calculating the closed form solutions of option price under stochastic volatility models using stochastic calculus and the Fourier inversion formula. In particular, we review and derive the option pricing formulas under Heston and correlated SteinStein models using a systematic and comprehensive approach which were derived individually earlier. We compare the empirical performances of the two stochastic volatility models and the Black-Scholes model in pricing KOSPI 200 index options.
\end{abstract}

\section{Introduction}

Based on no arbitrage arguments, Black and Scholes [3] and Merton [13] derived a partial differential equation for the value of European stock options. The Black-Scholes (B-S) model assumed that the asset price follows a geometric Brownian motion with a constant volatility. Because of its simplicity and analytical tractability, the B-S model is widely used among practitioners for pricing options. However, a number of empirical studies documented systematic abnormalities and biases about the asset returns and resulting option prices in the B-S framework. Among them, the most well-known bias of the $\mathrm{B}-\mathrm{S}$ model is the so-called volatility smile or skew. The implied volatilities from the market prices of options vary with respect to strike prices and maturities, contrary to the assumption of constant volatility in the B-S model. Empirical studies found that asset returns usually have a higher kurtosis and a heavy tail compared to the normal distribution which is assumed by the B-S model.

Received March 31, 2008

2000 Mathematics Subject Classification. Primary 91B28, 65C20.

Key words and phrases. option pricing, stochastic volatility model, Heston model, correlated Stein-Stein model, KOSPI 200 index option.

$\dagger$ This work was supported by the Korea Research Foundation Grant funded by the Korean Government (MOEHRD, Basic Research Promotion Fund) (KRF-2007-313-C00101).

$\ddagger$ This work was supported by the research fund (R0600842) of Seoul Development Institute.

(C)2009 The Korean Mathematical Society 
Another aspect of previous empirical finding is that asset returns and its volatility are negatively correlated although the effects on option pricing have been considered as a less crucial and primary issue in market and academia.

There have been abundant attempts to introduce new models to improve the restrictive B-S framework. Among the list, there are jump-diffusion models, CEV models, stochastic volatility models, local volatility models, and exponential Lévy models, which are by no means exhaustive yet and create an overwhelming list of references.

One of major directions is to relax the B-S assumption of deterministic volatility. To capture the volatility smile effect, it is assumed that the volatility is governed by a separate stochastic process which is termed as a stochastic volatility $(\mathrm{SV})$ model.

Various SV models have been studied in the literature such as Hull and White [8], Scott [18], Wiggins [21], Johnson and Shanno [9], Melino and Turnbull [12], Stein and Stein [20], Heston [7], and Nicolato and Venardos [15]. When dealing with option pricing models, it is important to have analytical solutions for pricing derivatives in a closed form. Among these SV models mentioned above, Stein-Stein model and Heston model are used more extensively. They admit analytic solutions and hence are computationally efficient. Between two SV models there are two distinct advantages which Heston's model can offer. Heston's model allows for nonnegative volatility and nonzero correlation between the asset price and volatility, while Stein and Stein's model doesn't.

Stein and Stein [20] assumed that the volatility follows a mean reverting Ornstein-Uhlenbeck process and presented explicit closed form solutions for asset price distributions and option prices. But its major weakness was the assumption of zero correlation between asset returns and its volatility. Heston [7] developed another stochastic volatility model, which follows a mean-reverting square-root process and asset returns and its volatility are correlated. A closed form solution for options was derived using an analytical expression of the characteristic function of log returns of underlying assets.

Scott [19] provided a jump diffusion model incorporating stochastic volatility and stochastic interest, each of which follows a mean-reverting square root processes such as Heston's volatility process. He derived a closed form solution for option price using stochastic calculus and the Fourier inversion formula. Schöbel and Zhu [17] applied the analogous technique as in Scott [19] to extend the Stein-Stein model to the case where asset return and its volatility are correlated. To be more precise, it was assumed in Schöbel and Zhu [17] that the volatility follows a mean-reverting Ornstein-Uhlenbeck process and is correlated with asset returns. Closed form solutions for option prices are obtained by employing the Fourier inversion formula.

In Bakshi, Cao, and Chen [1], they conducted a comprehensive empirical study on a relatively rich class of option pricing models which incorporate Heston's SV, stochastic interest rate, and Merton's random jumps which still 
admit option pricing formulas in a closed-form. As mentioned in their work, random jumps or stochastic interest rates added to SV model do not improve overall pricing performances and the SV model alone reduces pricing errors significantly, while the random-jump feature improves the fitness of short-term options and the stochastic interest rate feature improves the fit of long-term options. More recently Lin, Strong, and Xu [11], Zhang and Shu [22], Chen and Gau [4], and Dupoyet [6] investigated empirical performances of Heston's model on FTSE 100 index options, S\&P 500 index options and currency options respectively.

For pricing KOSPI 200 index options, Kim and Kim [10] examined four different classes of stochastic volatility models including GARCH model, Variance Gamma model, and Heston model. They found that the Heston model outperforms the other models for in-sample pricing, out-of-sample pricing, and hedging.

Another point of view for improving pricing performances is to stress the relevance of nonzero correlation between the asset return and its volatility. This phenomena was observed and examined earlier in Rubinstein [16], Bates [2] and Nandi [14]. In particular, Nandi [14] found substantial improvements in pricing out-of-the-money options and overall pricing performance allowing nonzero correlation in the empirical study for Heston's model.

The objective of this work is two-fold. The first is to suggest a unified way of deriving the closed form solutions for well-known and popular two versions of SV model using stochastic calculus and the Fourier inversion formula employed individually by Scott [19] and Schöbel and Zhu [17]. We derive the existing formulas for option prices under Heston and correlated Stein-Stein models in a unified method. For this purpose, we maintain our discussions in the present work to be self-contained to a great extent. The second is to examine two existing SV models and to analyze the empirical performances of the option prices on KOSPI 200 index between two SV models and the B-S model.

The paper is organized as follows: In Section 2 we describe the general settings of Heston and correlated Stein-Stein SV models. In Section 3 we derive and present the option pricing formulas for the two SV models in a comprehensive way. In Sections 4.1 and 4.2, we discuss the KOSPI 200 index options data and the results from parameter estimation for two SV models. In Section 4.3 we investigate model performances by analyzing out-of-sample pricing errors for two SV models and B-S model. Finally in Section 5 we summarize the results.

\section{The stochastic volatility models}

\subsection{The Heston model}

Let $\left\{S_{t}\right\}_{t \geq 0}$ denote the price of the underlying asset on a suitable probability space $(\Omega, \mathcal{F}, P)$ and satisfy the stochastic differential equation

$$
d S_{t}=\mu S_{t} d t+\sqrt{v_{t}} S_{t} d W_{t},
$$


where $v_{t}$ is a variance process and $\mu$ is a constant expected rate of return. We assume that $v_{t}$ follows Cox-Ingersoll-Ross (CIR) [5] process:

$$
d v_{t}=\kappa\left(\theta-v_{t}\right) d t+\sigma \sqrt{v_{t}} d Z_{t},
$$

where $\theta$ is a long-run average of $v_{t}, \kappa$ is a rate of mean reversion, $\sigma$ is called volatility of volatility. Here $\theta, \kappa, \sigma$ are constants and $W_{t}$ and $Z_{t}$ are two Brownian motions with a correlation coefficient $\rho \in[-1,1]$, i.e.,

$$
d W_{t} d Z_{t}=\rho d t .
$$

As in Heston [7], we choose the market price of the volatility risk proportional to the volatility. By shifting to a risk-neutral measure and applying Girsanov theorem to (1) and (2) we have the following equations under a risk neutral measure $Q$,

$$
\begin{aligned}
d S_{t} & =r S_{t} d t+\sqrt{v_{t}} S_{t} d W_{t}, \\
d v_{t} & =\kappa^{*}\left(\theta^{*}-v_{t}\right) d t+\sigma \sqrt{v_{t}} d Z_{t},
\end{aligned}
$$

where

$$
\kappa^{*}=\kappa+\lambda, \quad \theta^{*}=\frac{\kappa \theta}{\kappa+\lambda}, \quad d W_{t} d Z_{t}=\rho d t .
$$

The variance moves toward the long-run average variance $\theta^{*}$, with the speed determined by $\kappa^{*}$. We use the stochastic differential equation (3) for the evolutionary model of the underlying asset to describe Heston's model. It is convenient to write

$$
W_{t}=\rho Z_{t}+\sqrt{1-\rho^{2}} \hat{Z}_{t},
$$

where $\hat{Z}_{t}$ is a standard Brownian motion independent of $Z_{t}$.

\subsection{The correlated Stein-Stein model}

Let $\left\{S_{t}\right\}_{t \geq 0}$ denote the price of the underlying asset on a probability space $(\Omega, \mathcal{F}, P)$ and satisfy the stochastic differential equation (1). To simplify the notations, we use $u_{t}=\sqrt{v_{t}}$ for a volatility process and assume that $u_{t}$ follows a mean-reverting Ornstein-Uhlenbeck process,

$$
d u_{t}=\tilde{\kappa}\left(\tilde{\theta}-u_{t}\right) d t+\tilde{\sigma} d \tilde{Z}_{t}
$$

where $\tilde{\kappa}, \tilde{\theta}$ and $\tilde{\sigma}$ are constants.

As in Schöbel and Zhu [17], we consider the extension of the Stein-Stein model and assume that $W_{t}$ and $\tilde{Z}_{t}$ are correlated Brownian motions with a correlation coefficient $\tilde{\rho} \in[-1,1]$. Again following standard practice to take a risk-neutral measure $Q$, we assume that the asset price and volatility are governed as following two processes;

$$
\begin{aligned}
& d S_{t}=r S_{t} d t+u_{t} S_{t} d W_{t} \\
& d u_{t}=\tilde{\kappa}\left(\tilde{\theta}-u_{t}\right) d t+\tilde{\sigma} d \tilde{Z}_{t}
\end{aligned}
$$


Note that we continue to use the same parameters as in (5) to avoid displaying too many insignificant parameters. Here $W_{t}$ and $\tilde{Z}_{t}$ are correlated Brownian motions with a correlation coefficient $\tilde{\rho} \in[-1,1]$, i.e.,

$$
d W_{t} d \tilde{Z}_{t}=\tilde{\rho} d t .
$$

\section{The option pricing formulas}

For both SV models considered in the present work, the price of European call option can be expressed as a conditional expectation of a discounted payoff under the risk-neutral measure $Q$,

$$
E^{Q}\left[e^{-r \tau} \max (S(T)-K, 0) \mid \mathcal{F}_{t}\right],
$$

where $\tau \equiv T-t$ is the time to expiry, $r$ is the constant riskless interest rate, and $K$ is a given strike price. Here $\mathcal{F}_{t}$ is defined by the smallest $\sigma$-algebra generated by $\left\{W_{s}, Z_{s}: s \leq t\right\}$ for the Heston model and $\left\{W_{s}, \tilde{Z}_{s}: s \leq t\right\}$ for the correlated Stein-Stein model respectively.

In the present section, we investigate a systematic and unified method to provide closed-form solutions for European call option prices under two SV models. First, we consider the Heston's model under risk-neutral measure $Q$ and write

$$
\begin{aligned}
& C_{\text {Heston }}\left(S_{t}, v_{t}, T-t\right) \\
= & E^{Q}\left[e^{-r(T-t)} \max \left(S_{T}-K, 0\right) \mid \mathcal{F}_{t}\right] \\
= & E^{Q}\left[e^{-r(T-t)} S_{T} \mathbf{1}_{\left\{S_{T}>K\right\}} \mid \mathcal{F}_{t}\right]-K E^{Q}\left[e^{-r(T-t)} K \mathbf{1}_{\left\{S_{T}>K\right\}} \mid \mathcal{F}_{t}\right] \\
\equiv & C_{1}\left(S_{t}, v_{t}, T-t\right)-K C_{2}\left(S_{t}, v_{t}, T-t\right) .
\end{aligned}
$$

Again under the correlated Stein-Stein model governed by (6), we obtain following analogous expression for European call option price,

$$
\begin{aligned}
& C_{S-S}\left(S_{t}, u_{t}, T-t\right) \\
= & E^{Q}\left[e^{-r(T-t)} \max \left(S_{T}-K, 0\right) \mid \mathcal{F}_{t}\right] \\
= & E^{Q}\left[e^{-r(T-t)} S_{T} \mathbf{1}_{\left\{S_{T}>K\right\}} \mid \mathcal{F}_{t}\right]-K E^{Q}\left[e^{-r(T-t)} K \mathbf{1}_{\left\{S_{T}>K\right\}} \mid \mathcal{F}_{t}\right] \\
\equiv & \tilde{C}_{1}\left(S_{t}, u_{t}, T-t\right)-K \tilde{C}_{2}\left(S_{t}, u_{t}, T-t\right) .
\end{aligned}
$$

It is worthwhile to note that $C_{1}$ and $\tilde{C}_{1}$ stand for the values of asset-or-nothing call option for each SV model. Similarly, $C_{2}$ and $\tilde{C}_{2}$ denote the values of cash-or-nothing call option for each model.

\subsection{Asset-or-nothing options}

Let us denote the $\log$ of the asset price $x(t)=\ln S(t)$. For Heston's model, by change of the measure,

$$
\frac{d Q_{1}}{d Q}=e^{-r T} S_{T}
$$


we have

$$
\begin{aligned}
C_{1}\left(S_{t}, v_{t}, T-t\right) & =E^{Q}\left[e^{-r(T-t)} S_{T} \mathbf{1}_{\left\{x_{T}>\ln K\right\}} \mid \mathcal{F}_{t}\right] \\
& =E^{Q_{1}}\left[e^{r t} \mathbf{1}_{\left\{x_{T}>\ln K\right\}} \mid \mathcal{F}_{t}\right] E^{Q}\left[S_{T} e^{-r T} \mid \mathcal{F}_{t}\right] \\
& =E^{Q_{1}}\left[\mathbf{1}_{\left\{x_{T}>\ln K\right\}} \mid \mathcal{F}_{t}\right] S_{t} \\
& \equiv F_{1} S_{t} .
\end{aligned}
$$

The characteristic function $f_{1}$ of $x_{T}$ under $Q_{1}$ conditioned on $\mathcal{F}_{t}$ can be defined by

$$
\begin{aligned}
f_{1}(\phi) & =E^{Q_{1}}\left[e^{i \phi x_{T}} \mid \mathcal{F}_{t}\right] \\
& =\frac{E^{Q}\left[e^{i \phi x_{T}} S_{T} e^{-r T} \mid \mathcal{F}_{t}\right]}{E^{Q}\left[S_{T} e^{-r T} \mid \mathcal{F}_{t}\right]} \\
& =\frac{E^{Q}\left[e^{i \phi x_{T}} S_{T} e^{-r T} \mid \mathcal{F}_{t}\right]}{S_{t} e^{-r t}} \\
& =e^{-x_{t}-r(T-t)} E^{Q}\left[e^{(1+i \phi) x_{T}} \mid \mathcal{F}_{t}\right] .
\end{aligned}
$$

Consequently, by applying the Fourier inversion formula, we obtain

$$
F_{1}=\frac{1}{2}+\frac{1}{\pi} \int_{0}^{\infty} \operatorname{Re}\left(f_{1}(\phi) \frac{\exp (-i \phi \ln K)}{i \phi}\right) d \phi
$$

for Heston's model. Therefore, the asset-or-nothing call option can be computed by

$$
C_{1}\left(S_{t}, v_{t}, T-t\right)=S_{t} F_{1} .
$$

By repeating the same argument for the correlated Stein-Stein model, we have

$$
\widetilde{C}_{1}\left(S_{t}, u_{t}, T-t\right)=S_{t} \widetilde{F}_{1},
$$

where $\widetilde{F}_{1}$ and $\tilde{f}_{1}$ are defined analogously as in (10) and (11).

\subsection{Cash-or-nothing options}

The value of a cash-or-nothing call option for Heston's model can be expressed as the following,

$$
\begin{aligned}
C_{2}\left(S_{t}, v_{t}, T-t\right) & =E^{Q}\left[e^{-r(T-t)} \mathbf{1}_{\left\{x_{T}>\ln K\right\}} \mid \mathcal{F}_{t}\right] \\
& =e^{-r(T-t)} Q\left(x_{T}>\ln K \mid \mathcal{F}_{t}\right) \\
& \equiv e^{-r(T-t)} F_{2} .
\end{aligned}
$$

Now we calculate the characteristic function $f_{2}$ of $x_{T}$ under $Q$ conditioned on $\mathcal{F}_{t}$ by

$$
f_{2}(\phi)=E^{Q}\left[e^{i \phi x_{T}} \mid \mathcal{F}_{t}\right]
$$

Once we compute the characteristic function $f_{2}, F_{2}$ can be calculated using the Fourier inversion formula:

$$
F_{2}=\frac{1}{2}+\frac{1}{\pi} \int_{0}^{\infty} \operatorname{Re}\left(f_{2}(\phi) \frac{\exp (-i \phi \ln K)}{i \phi}\right) d \phi .
$$


Again for correlated Stein-Stein model, we write along the similar lines,

$$
\widetilde{C}_{2}\left(S_{t}, u_{t}, T-t\right)=e^{-r(T-t)} \widetilde{F}_{2},
$$

where $\widetilde{f}_{2}$ denotes the characteristic function of $x_{T}$ under $Q$ conditioned on $\mathcal{F}_{t}$, and $\widetilde{F}_{2}$ denotes the expression in $(16)$ with $\widetilde{f}_{2}$ replacing $f_{2}$.

\subsection{The option pricing formulas for the Heston model}

We now assume Heston's model, under which $S_{t}$ and $v_{t}$ follow the equations in (3) under $Q$. To derive the formulas for $f_{1}$ and $f_{2}$ in (10) and (15), it is convenient to obtain the Fourier transform of $x_{T}$ under $Q$ conditioned on $\mathcal{F}_{t}$ in a general setting. The proof of the lemma is given in Appendix.

Lemma 3.1. Assume that the value of the underlying asset price and its variance satisfy (3) under $Q$. Then, for $z=1+i \phi$ or $i \phi$ with $\phi$ real and $\tau=T-t$, we have

$$
\begin{aligned}
f(z)=E^{Q}\left[e^{z x_{T}} \mid \mathcal{F}_{t}\right]=\exp \left(z \left\{x_{t}+r \tau-\frac{\rho}{\sigma}\left(v_{t}\right.\right.\right. & \left.\left.\left.+\kappa^{*} \theta^{*} \tau\right)\right\}\right) \\
& \times \exp \left(A(\tau) v_{t}+B(\tau)\right),
\end{aligned}
$$

where the functions $A(\tau)$ and $B(\tau)$ satisfy

$$
A(\tau)=\frac{s_{2}\left(\gamma+\kappa^{*}\right)+s_{2} e^{\tau \gamma}\left(\gamma-\kappa^{*}\right)+2 s_{1}\left(e^{\tau \gamma}-1\right)}{-s_{2}\left(e^{\tau \gamma}-1\right) \sigma^{2}+\gamma-\kappa^{*}+e^{\tau \gamma}\left(\gamma+\kappa^{*}\right)}
$$

and

$$
B(\tau)=\frac{2 \theta^{*} \kappa^{*}}{\sigma^{2}} \ln \left[\frac{2 \gamma e^{\tau\left(\gamma+\kappa^{*}\right) / 2}}{-s_{2}\left(e^{\tau \gamma}-1\right) \sigma^{2}+\gamma-\kappa^{*}+e^{\tau \gamma}\left(\gamma+\kappa^{*}\right)}\right]
$$

with

$$
\begin{aligned}
& \gamma=\sqrt{\kappa^{* 2}-2 s_{1} \sigma^{2}}, \\
& s_{1}=z\left(\frac{\rho}{\sigma} \kappa^{*}-\frac{1}{2}\right)+\frac{1}{2} z^{2}\left(1-\rho^{2}\right), s_{2}=z \frac{\rho}{\sigma} .
\end{aligned}
$$

Once we compute the Fourier transform (18), we drive the value of European call option under the Heston SV model.

Theorem 3.2 (Heston Model). Assume that the underlying asset price and its variance satisfy (3) under $Q$. Then European call option pricing formula for the Heston model is given by

$$
C_{\text {Heston }}\left(S_{t}, v_{t}, T-t\right)=S_{t} F_{1}-e^{-r \tau} K F_{2},
$$

where $K$ is the strike price and $r$ is the riskless interest rate, and $\tau=T-t$ is the time to maturity. Here

$$
\begin{aligned}
f_{1}(\phi) & =f(1+i \phi) \exp \left(-x_{t}-r \tau\right) \\
f_{2}(\phi) & =f(i \phi) \\
F_{i} & =\frac{1}{2}+\frac{1}{\pi} \int_{0}^{\infty} \operatorname{Re}\left(f_{i}(\phi) \frac{\exp (-i \phi \ln K)}{i \phi}\right) d \phi,
\end{aligned}
$$

and $f$ is defined in (18). 


\subsection{The option pricing formulas for the correlated Stein-Stein model}

By employing the analogous techniques, we compute the characteristic functions for the correlated Stein-Stein model. The proof of the lemma is given in Appendix.

Lemma 3.3. Assume that the underlying asset price and its volatility satisfy (6) under $Q$. Then for $z=1+i \phi$ or $i \phi$ with $\phi$ real and $\tau=T-t$, we have

$$
\begin{aligned}
\tilde{f}(z)=E^{Q}\left[e^{z x_{T}} \mid \mathcal{F}_{t}\right]=\exp \left(z\left\{\left(x_{t}+r \tau\right)-\frac{1}{2} \tilde{\rho}\left\{\tilde{\sigma}^{-1} u_{t}^{2}+\tilde{\sigma} \tau\right\}\right\}\right) \\
\times \exp \left(\frac{1}{2} G(\tau) u_{t}^{2}+H(\tau) u_{t}+I(\tau)\right),
\end{aligned}
$$

where the functions $G(\tau), H(\tau)$ and $I(\tau)$ satisfy

$$
G(\tau)=\frac{1}{\tilde{\sigma}^{2}}\left(\tilde{\kappa}-\gamma_{1} \frac{\sinh \left(\gamma_{1} \tau\right)+\gamma_{2} \cosh \left(\gamma_{1} \tau\right)}{\cosh \left(\gamma_{1} \tau\right)+\gamma_{2} \sinh \left(\gamma_{1} \tau\right)}\right)
$$

and

$$
\begin{aligned}
H(\tau)= & \frac{1}{\tilde{\sigma}^{2} \gamma_{1}}\left(\frac{\left(\tilde{\kappa} \tilde{\theta} \gamma_{1}-\gamma_{2} \gamma_{3}\right)+\gamma_{3}\left(\sinh \left(\gamma_{1} \tau\right)+\gamma_{2} \cosh \left(\gamma_{1} \tau\right)\right)}{\cosh \left(\gamma_{1} \tau\right)+\gamma_{2} \sinh \left(\gamma_{1} \tau\right)}-\tilde{\kappa} \tilde{\theta} \gamma_{1}\right) \\
I(\tau)=-\frac{1}{2} \ln \left(\cosh \left(\gamma_{1} \tau\right)+\gamma_{2} \sinh \left(\gamma_{1} \tau\right)\right)+\frac{1}{2} \tilde{\kappa} \tau & \\
& +\frac{\tilde{\kappa}^{2} \tilde{\theta}^{2} \gamma_{1}^{2}-\gamma_{3}^{2}}{2 \tilde{\sigma}^{2} \gamma_{1}^{3}}\left(\frac{\sinh \left(\gamma_{1} \tau\right)}{\cosh \left(\gamma_{1} \tau\right)+\gamma_{2} \sinh \left(\gamma_{1} \tau\right)}-\gamma_{1} \tau\right) \\
& +\frac{\left(\tilde{\kappa} \tilde{\theta} \gamma_{1}-\gamma_{2} \gamma_{3}\right) \gamma_{3}}{\tilde{\sigma}^{2} \gamma_{1}^{3}}\left(\frac{\cosh \left(\gamma_{1} \tau\right)-1}{\cosh \left(\gamma_{1} \tau\right)+\gamma_{2} \sinh \left(\gamma_{1} \tau\right)}\right)
\end{aligned}
$$

with

$$
\begin{aligned}
& \gamma_{1}=\sqrt{\tilde{\kappa}^{2}+2 s_{1} \tilde{\sigma}^{2}}, \gamma_{2}=\frac{1}{\gamma_{1}}\left(\tilde{\kappa}-2 s_{3} \tilde{\sigma}^{2}\right), \gamma_{3}=\tilde{\kappa}^{2} \tilde{\theta}-s_{2} \tilde{\sigma}^{2} \\
& s_{1}=-\frac{1}{2} z^{2}\left(1-\tilde{\rho}^{2}\right)+\frac{1}{2} z\left(1-2 \tilde{\kappa} \tilde{\rho} \tilde{\sigma}^{-1}\right) \\
& s_{2}=z \tilde{\kappa} \tilde{\theta} \tilde{\rho} \tilde{\sigma}^{-1} \\
& s_{3}=\frac{1}{2} z \tilde{\rho} \tilde{\sigma}^{-1} .
\end{aligned}
$$

Using Lemma 3.3, we can similarly obtain the value of European call option under the correlated Stein-Stein model as follows:

Theorem 3.4 (Correlated Stein-Stein Model). Assume that the underlying asset price and its volatility satisfy (6) under Q. Then European call option pricing formula for the correlated Stein-Stein model is given by

$$
\widetilde{C}_{S-S}\left(S_{t}, u_{t}, T-t\right)=S_{t} \widetilde{F}_{1}-e^{-r \tau} K \widetilde{F}_{2},
$$


where $K$ is the strike price and $r$ is the riskless interest rate, and $\tau=T-t$ is the time to maturity. Here

$$
\begin{aligned}
\tilde{f}_{1}(\phi) & =\widetilde{f}(1+i \phi) \exp \left(-x_{t}-r \tau\right) \\
\widetilde{f}_{2}(\phi) & =\widetilde{f}(i \phi) \\
\widetilde{F}_{i} & =\frac{1}{2}+\frac{1}{\pi} \int_{0}^{\infty} \operatorname{Re}\left(\widetilde{f}_{i}(\phi) \frac{\exp (-i \phi \ln K)}{i \phi}\right) d \phi
\end{aligned}
$$

and $\tilde{f}$ is defined in $(21)$.

\section{Empirical performances on KOSPI200 index options}

Based on the closed form solutions presented in Theorem 3.2 for the Heston model and in Theorem 3.4 for the correlated Stein-Stein model we examine and compare the relative empirical performances of two SV models and B-S model in pricing KOSPI 200 options. To perform the empirical test, it is essential to estimate the unobservable structure parameters and the spot volatility using an efficient methodology. We use the most popular approach of minimizing the weighted sum of squared pricing errors between the models prices and market prices for estimation of parameters. We assess and analyze two distinct types of out-of-sample pricing errors for KOSPI 200 options under two SV models and B-S model.

\subsection{Data description}

The KOSPI200 (Korea Stock Price Index 200) option was developed at the Korea Stock Exchange (KSE) in June 1994 by selecting 200 stocks from a broad range of industry groups of stocks listed on the KSE. Our empirical study is based on European style KOSPI 200 options over the period from Jan. 2, 2004 through Sept. 21, 2006. For each day in the sample, the last reported bidask price quotes and closing prices are recorded which are used for different purposes.

To enhance the efficiency of our analysis, we eliminate options prices from our data to avoid unnecessary bias and noises. We exclude options with maturities less than 6 days or more than 90 days, and options with prices lower than 0.2 . Moreover options with the moneyness $S / K$ is less than 0.8 or larger than 1.2, i.e., very deep out-of-money or deep in-the-money are discarded. The prices which are not satisfying the arbitrage restriction are excluded, i.e.,

$$
C^{\text {Market }} \geq S-D-e^{-r(T-t)} K
$$

where $C^{\text {Market }}$ is the observed market prices and $D$ is a present value of a sum of the future dividends during remaining time $T-t$. Following the convention of $\mathrm{KSE}$, the 91-day certificate of deposit (CD 91-day) rate is used as the risk-free interest rate.

Through a series of data filtering, a total of 17,843 call options over the period 879 days are recorded. We categorize the option data according to their 
moneyness $S / K$ and maturity and describe their characteristics in Table 1 . On the basis of moneyness option data consist of 32.9\% OTM, 32.4\% ATM, and $34.7 \%$ ITM options. The study shows that the average call option price ranges from 0.4077 for short-term and deep OTM options to 13.5873 for short-term and deep ITM call options. In turns out that on average we retain about 26 call option prices observed on each day.

TABLE 1. KOSPI200 Call Option Data

\begin{tabular}{|c|c|c|c|c|c|}
\hline & \multirow[b]{2}{*}{ Moneyness } & \multicolumn{3}{|c|}{ Days-to-Maturity } & \multirow[b]{2}{*}{ Overall } \\
\hline & & $\leq 30$ & $31-60$ & $61-90$ & \\
\hline \multirow[t]{6}{*}{ OTM } & $S / K<0.94$ & 0.4077 & 0.6688 & 1.1459 & 0.8640 \\
\hline & & $(0.0083)$ & $(0.0157)$ & $(0.0248)$ & $(0.0224)$ \\
\hline & & $\{227\}$ & $\{1398\}$ & $\{1335\}$ & $\{2960\}$ \\
\hline & $0.94 \leq S / K<0.97$ & 0.6924 & 1.5706 & 2.5822 & 1.7139 \\
\hline & & $(0.0163)$ & $(0.0275)$ & $(0.0339)$ & $(0.0392)$ \\
\hline & & $\{687\}$ & $\{1218\}$ & $\{1009\}$ & $\{2914\}$ \\
\hline \multirow[t]{6}{*}{ ATM } & $0.97 \leq S / K<1.00$ & 1.6296 & 3.1394 & 4.3130 & 3.0389 \\
\hline & & $(0.0328)$ & $(0.0406)$ & $(0.0452)$ & $(0.0569)$ \\
\hline & & $\{931\}$ & $\{1163\}$ & $\{938\}$ & $\{3032\}$ \\
\hline & $1.00 \leq S / K<1.03$ & 3.7496 & 5.2555 & 6.3118 & 5.0725 \\
\hline & & $(0.0485)$ & $(0.0543)$ & $(0.0588)$ & $(0.0662)$ \\
\hline & & $\{876\}$ & $\{1091\}$ & $\{774\}$ & $\{2741\}$ \\
\hline \multirow[t]{9}{*}{ ITM } & $1.03 \leq S / K<1.06$ & 6.7828 & 7.8816 & 8.8208 & 7.7879 \\
\hline & & $(0.0665)$ & $(0.0723)$ & $(0.0746)$ & $(0.0772)$ \\
\hline & & $\{735\}$ & $\{972\}$ & $\{627\}$ & $\{2334\}$ \\
\hline & $S / K \geq 1.06$ & 14.5470 & 13.3575 & 13.5873 & 13.7584 \\
\hline & & $(0.2164)$ & $(0.1600)$ & $(0.1427)$ & $(0.1745)$ \\
\hline & & $\{1089\}$ & $\{1673\}$ & $\{1100\}$ & $\{3862\}$ \\
\hline & Overall & 5.7639 & 5.6209 & 5.8003 & 5.7155 \\
\hline & & $(0.2330)$ & $(0.1997)$ & $(0.1862)$ & $(0.2046)$ \\
\hline & & $\{4545\}$ & $\{7515\}$ & $\{5783\}$ & $\{17843\}$ \\
\hline
\end{tabular}

This table reports average option price, standard error (in parentheses), and the number of options (in braces), for each moneyness-maturity category. The sample period extends from January 2, 2004 to September 21, 2006. Daily information from the last transaction prices of each option contract is used to obtain the summary statistics. Moneyness is defined as $S / K$, where $S$ denotes the spot price and $K$ denotes the strike price. OTM, ATM, and ITM denote out-of-the money, at-themoney, and in-the-money options, respectively.

\subsection{Parameter estimation}

To compute option prices for Heston's model, one needs to estimate the vector of spot variances $v_{t_{0}}$ and structural parameters, $\Phi=\left(\kappa^{*}, \theta^{*}, \sigma, \rho, v_{t_{0}}\right)$. 
TABLE 2. Parameter Estimation

\begin{tabular}{ccccccc}
\hline Model & \multicolumn{5}{c}{ Parameters } \\
\hline \hline B-S & $\sigma_{i m p}$ & & & & & \\
& 0.1993 & & & & & \\
& $(0.0015)$ & & & & & \\
\hline \multirow{2}{*}{ S-S } & & $\widetilde{\kappa}$ & $\widetilde{\theta}$ & $\widetilde{\sigma}$ & $\widetilde{\rho}$ & $u_{t_{0}}$ \\
\cline { 2 - 6 } & & 2.5214 & 0.1717 & 0.2098 & -0.1830 & 0.1998 \\
& $(0.1646)$ & $(0.0075)$ & $(0.0082)$ & $(0.0189)$ & $(0.0021)$ \\
\hline Heston & & $\kappa^{*}$ & $\theta^{*}$ & $\sigma$ & $\rho$ & $v_{t_{0}}$ \\
& & 6.9282 & 0.0698 & 0.2896 & -0.3496 & 0.0427 \\
& $(0.2765)$ & $(0.0038)$ & $(0.0115)$ & $(0.0246)$ & $(0.0009)$ \\
\hline
\end{tabular}

This table reports the daily average and its standard error (in parentheses) of the estimated parameters. The Black-Scholes model in which a single implied volatility is estimated across all strikes and maturities on a given day and the SV models in which parameters are estimated by minimizing the weighted sum of squared pricing errors between model and market option prices for each day.

For the counterpart of Stein-Stein model, the vector of the spot volatility $u_{t_{0}}$, and structural parameters, $\tilde{\Phi}=\left(\tilde{\kappa}, \tilde{\theta}, \tilde{\sigma}, \tilde{\rho}, u_{t_{0}}\right)$, are input parameters to be estimated from option data. Following the standard practice by most researchers and practitioners, we use the least-square-minimization method to obtain the parameters implied by option data.

We describe the estimation procedure as follows. First, we collect $N_{t}$ option values on the same underlying asset in the same day $t$. These options have different times to maturity and strike prices. Let $C_{i, t}^{\mathrm{Mid}}$ be the mid price of bid-ask spread of the $i$-th option on day $t$, and $C_{i, t}^{\text {Model }}$ be either the Heston's or the correlated Stein-Stein model prices of the $i$-th option on day $t$. The parameter set $\Phi$ and $\tilde{\Phi}$ are then determined by

$$
\min \sum_{i=1}^{N_{t}} \omega_{i}\left(C_{i, t}^{\mathrm{Mid}}-C_{i, t}^{\mathrm{Model}}\right)^{2} \quad \text { for } t=1,2, \ldots, T_{d},
$$

where $T_{d}$ is the total number of days in the sample. Here we take the observed mid price $C_{i, t}^{\mathrm{Mid}}$ and the weight function $\omega_{i}$ as following

$$
C_{i, t}^{\mathrm{Mid}}=\frac{C_{i, t}^{\mathrm{bid}}+C_{i, t}^{\mathrm{ask}}}{2}, \omega_{i}=\frac{1}{C_{i, t}^{\mathrm{ask}}-C_{i, t}^{\mathrm{bid}}}
$$

where $C_{i, t}^{\text {bid }}$ and $C_{i, t}^{\text {ask }}$ are the bid and ask prices of the $i$-th option at the day $t$. If the bid-ask spread of a particular option is great, then we have a wider range of market prices around the mid-price which necessarily imply small liquidity as a result. It is quite reasonable to take less weights for such options in estimating the implied parameters. 
For the Heston and the correlated Stein-Stein models, Table 2 reports the averages and the standard errors (in parentheses) of the parameters, which are estimated daily. The implied parameters of KOSPI200 index call option in the sample are shown to be the average values of $\left\{\kappa^{*}, \theta^{*}, \sigma\right\}=\{6.9282,0.0698$, $0.2896\}$ for the Heston model and $\{\tilde{\kappa}, \tilde{\theta}, \tilde{\sigma}\}=\{2.5214,0.1717,0.2098\}$ for the correlated Stein-Stein model. The average of estimated correlation coefficients are $\rho=-0.3496$ and $\tilde{\rho}=-0.1830$. We conform the widely accepted assumption that asset returns and volatility are negatively correlated, as documented by many previous results such as Rubinstein [16], Nandi [14], and Bates [2].

\subsection{Empirical tests}

Using estimation methology described earlier, we perform out-of-sample pricing tests for two versions of SV model and the B-S model. For two SV models, the implied structural parameters and spot variance and spot volatility are computed by minimizing the weighted sum of squared errors between model prices and mid-prices from the previous day while for B-S model, an average single implied volatility is computed from the previous day.

For measurement of pricing errors, we demonstrate values of the mean percentage pricing error(MPE) and mean absolute pricing error(MAE) defined as

(1) MPE

$$
\frac{1}{N} \sum_{i=1}^{N}\left(\frac{C_{i}^{\text {Market }}-C_{i}^{\text {Model }}}{C_{i}^{\text {Market }}}\right)
$$

(2) MAE

$$
\frac{1}{N} \sum_{i=1}^{N}\left|C_{i}^{\text {Market }}-C_{i}^{\text {Model }}\right|
$$

where $N$ is the total number of options in a particular moneyness-maturity category, $C_{i}^{\text {Market }}$ and $C_{i}^{\text {Model }}$ represent the market price and the theoretical model price, respectively. These measurements were popular, e.g., in Bakshi, Cao, and Chen [1].

Table 3 and Table 4 report the MPE's and MAE's of the Black-Scholes model, the correlated Stein-Stein model, the Heston model, for each moneynessmaturity categories. We summarize the results and draw some conclusions. From both pricing error measurements, two SV models improve pricing performances significantly over B-S model which is not surprising as was found by Bakshi, Cao, and Chen [1]. In comparison of two SV models, it is worthwhile to notice that Heston model performs slightly better than correlated Stein-Stein model in the most categories, although two SV models have the identical number of structural parameters. Pricing improvements of two SV models over B-S model are particularly noticeable for OTM options in pricing error measurements. As an example, for long-term option with moneyness less than 0.94, MPE for B-S, Stein-Stein and Heston models are $-2.76 \%,-1.33 \%,-1.05 \%$ and 
TABle 3. The Out-of-sample Mean Percentage Pricing Error (MPE)

\begin{tabular}{|c|c|c|c|c|c|c|}
\hline & \multirow[b]{2}{*}{ Moneyness } & & \multicolumn{3}{|c|}{ Days-to-Maturity } & \multirow[b]{2}{*}{ Overall } \\
\hline & & & $\leq 30$ & $31-60$ & $61-90$ & \\
\hline \multirow[t]{11}{*}{ OTM } & \multirow[t]{5}{*}{$S / K<0.94$} & $\mathrm{~B}-\mathrm{S}$ & $\begin{array}{c}-0.0923 \\
(0.0123)\end{array}$ & $\left.\begin{array}{c}-0.2427 \\
(0.0164\end{array}\right)$ & $\begin{array}{c}-0.2276 \\
(0.0132)\end{array}$ & $\begin{array}{c}-0.2243 \\
(0.0148\end{array}$ \\
\hline & & $\mathrm{S}-\mathrm{S}$ & -0.0956 & -0.0367 & -0.0133 & -0.0307 \\
\hline & & & $(0.0044)$ & $(0.0037)$ & $(0.0054)$ & $(0.0047)$ \\
\hline & & Heston & -0.0808 & -0.0148 & -0.0105 & -0.0179 \\
\hline & & & $(0.0043)$ & $(0.0032)$ & $(0.0054)$ & $(0.0044)$ \\
\hline & \multirow[t]{6}{*}{$0.94 \leq S / K<0.97$} & B-S & -0.1386 & -0.1318 & -0.1072 & -0.1249 \\
\hline & & & $(0.0138)$ & $(0.0118)$ & $(0.0094)$ & $(0.0116)$ \\
\hline & & S-S & -0.0591 & -0.0042 & 0.0119 & -0.0116 \\
\hline & & & $(0.0044)$ & $(0.0027)$ & $(0.0036)$ & $(0.0036)$ \\
\hline & & Heston & -0.0333 & 0.0050 & 0.0049 & -0.0041 \\
\hline & & & $(0.0042)$ & $(0.0020)$ & $(0.0035)$ & $(0.0033)$ \\
\hline \multirow[t]{12}{*}{ ATM } & \multirow[t]{6}{*}{$0.97 \leq S / K<1.00$} & B-S & -0.0865 & -0.0446 & -0.0586 & -0.0618 \\
\hline & & & $(0.0103)$ & $(0.0056)$ & $(0.0059)$ & $(0.0075)$ \\
\hline & & S-S & -0.0052 & 0.0214 & 0.0146 & 0.0111 \\
\hline & & & $(0.0021)$ & $(0.0017)$ & $(0.0034)$ & $(0.0025)$ \\
\hline & & Heston & -0.0008 & 0.0164 & 0.0049 & 0.0076 \\
\hline & & & $(0.0021)$ & $(0.0016)$ & $(0.0032)$ & $(0.0024)$ \\
\hline & \multirow[t]{6}{*}{$1.00 \leq S / K<1.03$} & B-S & -0.0209 & -0.0305 & -0.0532 & -0.0338 \\
\hline & & & $(0.0037)$ & $(0.0035)$ & $(0.0051)$ & $(0.0041)$ \\
\hline & & S-S & -0.0012 & 0.0011 & -0.0126 & -0.0035 \\
\hline & & & $(0.0015)$ & $(0.0018)$ & $(0.0041)$ & $(0.0026)$ \\
\hline & & Heston & -0.0022 & -0.0024 & -0.0181 & -0.0068 \\
\hline & & & $(0.0016)$ & $(0.0018)$ & $(0.0040)$ & $(0.0026)$ \\
\hline \multirow[t]{18}{*}{ ITM } & \multirow[t]{6}{*}{$1.03 \leq S / K<1.06$} & B-S & -0.0090 & -0.0269 & -0.0484 & -0.0270 \\
\hline & & & $(0.0027)$ & $(0.0030)$ & $(0.0039)$ & $(0.0032)$ \\
\hline & & S-S & -0.0126 & -0.0180 & -0.0289 & -0.0192 \\
\hline & & & $(0.0023)$ & $(0.0023)$ & $(0.0035)$ & $(0.0027)$ \\
\hline & & Heston & -0.0123 & -0.0176 & -0.0294 & -0.0191 \\
\hline & & & $(0.0023)$ & $(0.0023)$ & $(0.0034)$ & $(0.0027)$ \\
\hline & \multirow[t]{6}{*}{$S / K \geq 1.06$} & B-S & -0.0003 & -0.0217 & -0.0430 & -0.0217 \\
\hline & & & $(0.0020)$ & $(0.0027)$ & $(0.0029)$ & $(0.0026)$ \\
\hline & & S-S & -0.0038 & -0.0233 & -0.0391 & -0.0223 \\
\hline & & & $(0.0020)$ & $(0.0024)$ & $(0.0024)$ & $(0.0024)$ \\
\hline & & Heston & -0.0036 & -0.0222 & -0.0376 & -0.0214 \\
\hline & & & $(0.0020)$ & $(0.0025)$ & $(0.0024)$ & $(0.0024)$ \\
\hline & \multirow[t]{6}{*}{ Overall } & B-S & -0.0489 & -0.0861 & -0.1013 & -0.0816 \\
\hline & & & $(0.0082)$ & $(0.0096)$ & $(0.0087)$ & $(0.0090)$ \\
\hline & & S-S & -0.0180 & -0.0115 & -0.0109 & -0.0130 \\
\hline & & & $(0.0028)$ & $(0.0027)$ & $(0.0040)$ & $(0.0032)$ \\
\hline & & Heston & -0.0125 & -0.0070 & -0.0135 & -0.0105 \\
\hline & & & $(0.0027)$ & $(0.0024)$ & $(0.0039)$ & $(0.0030)$ \\
\hline
\end{tabular}

The Black-Scholes model price is computed by using the average implied volatility from the previous day. The SV model prices are computed by using the implied parameters estimated from minimizing the weighted sum of squared errors between the mid price and the model price from the previous day. The reported percentage pricing error is the sample average of the market price minus the model price, divided by the market price. The numbers in the parentheses are the standard errors.

MAE for B-S, Stein-Stein and Heston models are 0.2592, 0.0991, and 0.0966 respectively. By examining MPE, we find that regardless of pricing models, OTM options are overpriced in each maturity class, whereas the magnitude of errors in two SV models is much smaller than in B-S model. On the basis of 
TABle 4. The Out-of-sample Mean Absolute Pricing Error(MAE)

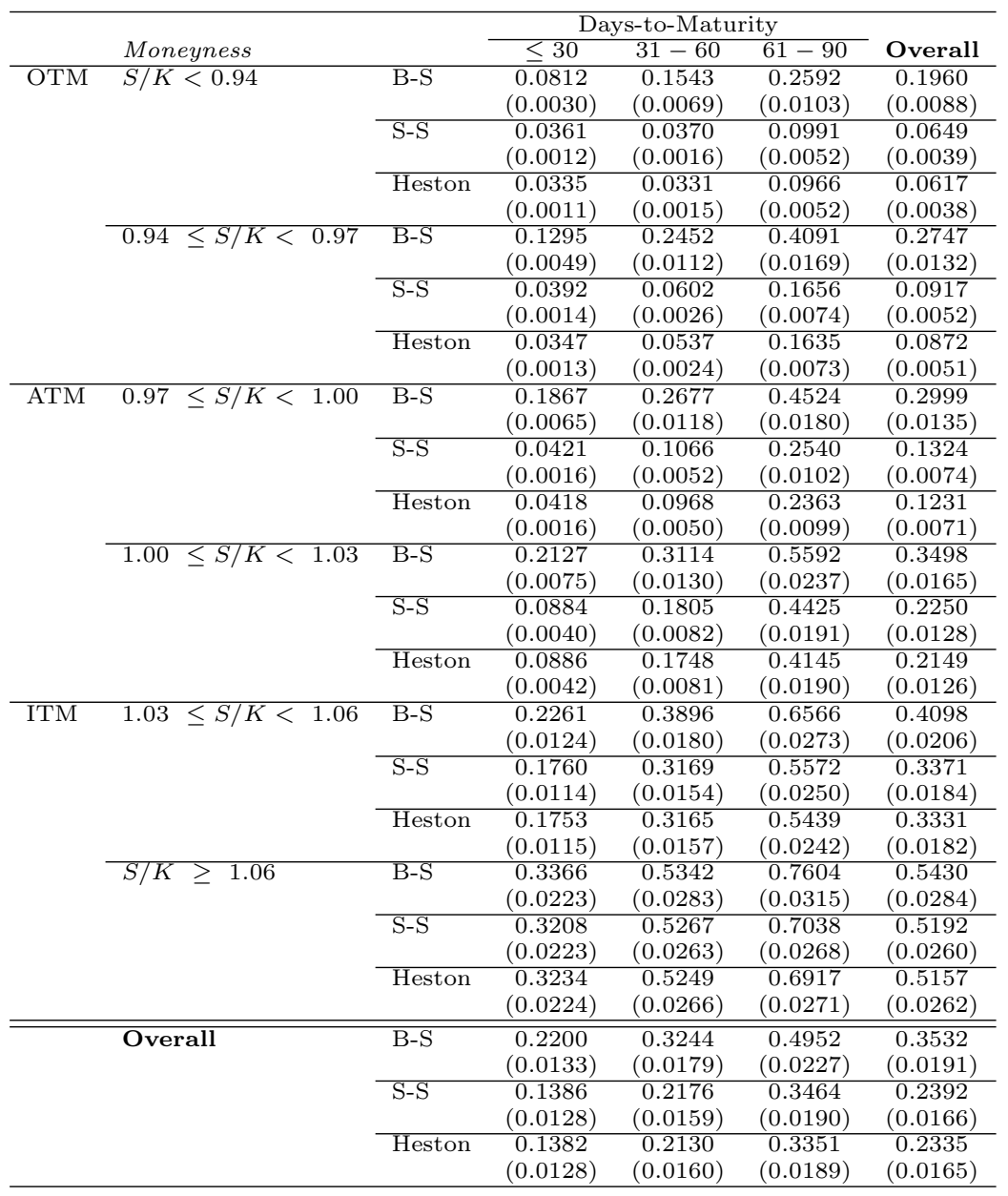

The Black-Scholes model price is computed by using the average implied volatility from the previous day. The SV model prices are computed by using the implied parameters estimated from minimizing the weighted sum of squared errors between the mid price and the model price from the previous day. The reported absolute pricing error is the absolute value of the market price minus the model price within each moneyness-maturity category. The numbers in the parentheses are the standard errors.

MPE, the pricing improvements by two SV models over B-S model decrease as moneyness increases, i.e., from OTM to ATM and from ATM to ITM in each maturity group. Contrary to this, the magnitude of absolute pricing errors 
reported in MAE typically increase as the moneyness increase in each maturity class across all models, since the options become more valuable as the moneyness increases. We observe again that in MAE measurement, the Heston model outperforms the other models in most of the moneyness-maturity groups, and the magnitude of improvement is more substantial for OTM options than for ITM options.

\section{Conclusions}

In this work, we review and derive the closed form solutions for the values of European call options under the Heston's and the correlated Stein-Stein stochastic volatility models using a unified approach. The analytic solutions are derived using stochastic calculus and Fourier inversion formula. Pricing performances of two versions of SV models and B-S model are empirically analyzed using the KOSPI 200 index option data from Jan. 2, 2004 through Sept. 21, 2006. The cross-sectional implied parameters are estimated from minimizing the weighted sum of squared errors between the market and the model prices for each model. Through the measurements of pricing errors, we conclude that two SV models outperform significantly over B-S model and the Heston model performs slightly better than the correlated Stein-Stein model.

\section{Appendix}

In this section, we prove the formulas of the characteristic functions (18) and (21) for the Heston model in Lemma 3.1 and the correlated Stein-Stein model in Lemma 3.3 respectively.

\section{Proof of the Lemma 3.1}

We adopt the approach employed in Scott [19] and provide more detailed discussions along the lines. Let us denote the log of the stock price $x_{t}=\ln S(t)$. Then from the stochastic differential equation $(3), x_{t}$ satisfies

$$
d x_{t}=\left(r-\frac{1}{2} v_{t}\right) d t+\sqrt{v_{t}} d W_{t} .
$$

For $z=1+i \phi$, we obtain

$$
\begin{aligned}
f(z) & =f(1+i \phi) \\
& =E^{Q}\left[e^{(1+i \phi) x_{T}} \mid \mathcal{F}_{t}\right] \\
& =e^{(1+i \phi) x_{t}} E^{Q}\left[e^{(1+i \phi)\left(x_{T}-x_{t}\right)} \mid \mathcal{F}_{t}\right] \\
& =e^{(1+i \phi)\left(x_{t}+r \tau\right)} E^{Q}\left[e^{(1+i \phi)\left(\int_{t}^{T}\left(-\frac{1}{2} v_{s}\right) d s+\int_{t}^{T} \sqrt{v_{s}} d W_{s}\right)} \mid \mathcal{F}_{t}\right] .
\end{aligned}
$$

Using the relation (4), we can write

$$
\int_{t}^{T} \sqrt{v_{s}} d W_{s}=\int_{t}^{T} \sqrt{v_{s}}\left(\rho d Z_{s}+\sqrt{1-\rho^{2}} d \hat{Z}_{s}\right)
$$


where $Z_{s}$ and $\hat{Z}_{s}$ are independent Brownian motions. We introduce another filtration $\widehat{\mathcal{F}}_{t}$, the smallest $\sigma$-algebra generated by $\left\{W_{s}, s \leq t, Z_{u}, u \leq T\right\}$. Then the expectation in $(26)$ can be written

$$
\begin{aligned}
E^{Q}\left[E^{Q}\left[\exp \left(-\frac{1}{2}(1+i \phi) \int_{t}^{T} v_{s} d s\right) \exp \left((1+i \phi) \int_{t}^{T} \sqrt{v_{s}} d W_{s}\right) \mid \widehat{\mathcal{F}}_{t}\right] \mid \mathcal{F}_{t}\right] \\
=E^{Q}\left[\exp \left(-\frac{1}{2}(1+i \phi) \int_{t}^{T} v_{s} d s\right) \exp \left((1+i \phi) \rho \int_{t}^{T} \sqrt{v_{s}} d Z_{s}\right)\right. \\
\left.E^{Q}\left[\exp \left((1+i \phi) \sqrt{1-\rho^{2}} \int_{t}^{T} \sqrt{v_{s}} d \hat{Z}_{s}\right) \mid \widehat{\mathcal{F}}_{t}\right] \mid \mathcal{F}_{t}\right] .
\end{aligned}
$$

Since

we obtain

$$
\int_{t}^{T} \sqrt{1-\rho^{2}} \sqrt{v_{s}} d \hat{Z}_{s} \sim \mathcal{N}\left(0, \int_{t}^{T}\left(1-\rho^{2}\right) v_{s} d s\right)
$$

$$
\begin{aligned}
& E^{Q}\left[\exp \left((1+i \phi) \sqrt{1-\rho^{2}} \int_{t}^{T} \sqrt{v_{s}} d \hat{Z}_{s}\right) \mid \widehat{\mathcal{F}}_{t}\right] \\
= & \exp \left(\frac{1}{2}(1+i \phi)^{2}\left(1-\rho^{2}\right) \int_{t}^{T} v_{s} d s\right) .
\end{aligned}
$$

Also from (3), we have

$$
\begin{aligned}
& (1+i \phi) \rho \int_{t}^{T} \sqrt{v_{s}} d Z_{s} \\
= & (1+i \phi) \frac{\rho}{\sigma}\left(\int_{t}^{T} d v_{s}-\int_{t}^{T} k^{*}\left(\theta^{*}-v_{s}\right) d s\right) \\
= & (1+i \phi) \frac{\rho}{\sigma}\left(v_{T}-v_{t}-k^{*} \theta^{*} \tau\right)+(1+i \phi) \frac{k^{*} \rho}{\sigma} \int_{t}^{T} v_{s} d s .
\end{aligned}
$$

As a consequence, we have

$$
f(1+i \phi)=e^{(1+i \phi)\left(x_{t}+r \tau\right)-(1+i \phi) \frac{\rho}{\sigma}\left(v_{t}+\kappa^{*} \theta^{*} \tau\right)} E^{Q}\left[e^{s_{2} v_{T}} e^{s_{1} \int_{t}^{T} v_{s} d s} \mid \mathcal{F}_{t}\right],
$$

where

$$
s_{1}=(1+i \phi)\left(\frac{k^{*} \rho}{\sigma}-\frac{1}{2}\right)+\frac{1}{2}(1+i \phi)^{2}\left(1-\rho^{2}\right), \quad s_{2}=(1+i \phi) \frac{\rho}{\sigma} .
$$

Let us define

$$
y(v, t, T)=E^{Q}\left[e^{s_{2} v(T)} e^{s_{1} \int_{t}^{T} v_{s} d s} \mid \mathcal{F}_{t}\right],
$$

with $s_{1}$ and $s_{2}$ are defined in (28). Then the Feynman-Kac stochastic representation theorem provides us that $y$ is the solution of the following PDE

$$
\frac{\partial y}{\partial t}+k^{*}\left(\theta^{*}-v\right) \frac{\partial y}{\partial v}+\frac{1}{2} \sigma^{2} v \frac{\partial^{2} y}{\partial v^{2}}+s_{1} v y=0
$$


with a final condition

$$
y(v, T, T)=e^{s_{2} v} .
$$

By analogy with Black-Scholes formula, we guess a solution of the form:

$$
y(v, t, T)=e^{A(T-t) v+B(T-t)} .
$$

By substituting the proposed value in (29), we obtain the following ordinary differential equations (ODEs) for $A(\tau)$ and $B(\tau)$ :

$$
\begin{aligned}
& A^{\prime}(\tau)=k^{*} A(\tau)-\frac{1}{2} \sigma^{2} A^{2}(\tau)-s_{1}, \quad A(0)=s_{2}, \\
& B^{\prime}(\tau)=-k^{*} \theta^{*} A(\tau), \quad B(0)=0 .
\end{aligned}
$$

The solutions of the above Riccati equation have well known formula from the literature. Hence $f(1+i \phi)$ is given by

$$
f(1+i \phi)=e^{(1+i \phi)\left(x_{t}+r \tau\right)-(1+i \phi) \frac{\rho}{\sigma}\left(v_{t}+\kappa^{*} \theta^{*} \tau\right)} e^{A(\tau) v_{t}+B(\tau)},
$$

where $\tau=T-t$ and $A(\tau)$ and $B(\tau)$ are defined in (19) and (20) respectively for $z=1+i \phi$. For $z=i \phi$, one can apply similar techniques to get the expression in (18) for $f(i \phi)$. This concludes the lemma.

The similar calculations are performed for the correlated Stein-Stein model to get Lemma 3.3.

\section{Proof of the Lemma 3.3}

We follow the proof given in Shöbel and Zhu [17]. Let us denote the log of the stock price $x_{t}=\ln S_{t}$. Then from the stochastic differential equation (6), $x_{t}$ satisfies

$$
d x_{t}=\left(r-\frac{1}{2} u_{t}^{2}\right) d t+u_{t} d W_{t}
$$

For $z=1+i \phi$, we obtain

$$
\begin{aligned}
\tilde{f}(z) & =\tilde{f}(1+i \phi) \\
& =E^{\widetilde{Q}}\left[e^{\left.(1+i \phi) x_{T}\right)} \mid \mathcal{F}_{t}\right] \\
& =e^{(1+i \phi)\left(x_{t}+r \tau\right)} E^{\widetilde{Q}}\left[e^{\left\{(1+i \phi) \int_{t}^{T}\left(-\frac{1}{2} u_{s}^{2}\right) d s+(1+i \phi) \int_{t}^{T} u_{s} d W_{s}\right\}} \mid \mathcal{F}_{t}\right]
\end{aligned}
$$

From Ito's formula and the equation (6), we have

$$
\int_{t}^{T} u_{s} d \tilde{Z}_{s}=\frac{1}{2 \tilde{\sigma}}\left\{u_{T}^{2}-u_{t}^{2}-\tilde{\sigma}^{2}(T-t)-2 \tilde{\kappa} \int_{t}^{T} u_{s}\left(\tilde{\theta}-u_{s}\right) d s\right\} .
$$

We perform similar calculations as in the proof of the Lemma 3.1 and to obtain

$$
\tilde{f}(1+i \phi)=e^{\left.(1+i \phi)\left(x_{t}+r \tau\right)-\frac{1}{2}(1+i \phi) \tilde{\rho}\left(\tilde{\sigma} \tau+u_{t}^{2} \tilde{\sigma}^{-1}\right)\right\}} E^{\widetilde{Q}}\left[e^{s_{3} u_{T}^{2}-\int_{t}^{T}\left(s_{1} u_{s}^{2}+s_{2} u_{s}\right) d s} \mid \mathcal{F}_{t}\right]
$$


where

$$
\begin{aligned}
& s_{1}=-\frac{1}{2}(1+i \phi)^{2}\left(1-\tilde{\rho}^{2}\right)+\frac{1}{2}(1+i \phi)\left(1-2 \tilde{\kappa} \tilde{\rho} \tilde{\sigma}^{-1}\right), \\
& s_{2}=(1+i \phi) \tilde{\kappa} \tilde{\theta} \tilde{\rho} \tilde{\sigma}^{-1}, s_{3}=\frac{1}{2}(1+i \phi) \tilde{\rho} \tilde{\sigma}^{-1} .
\end{aligned}
$$

Let us define

$$
y(u, t, T)=E^{\widetilde{Q}}\left[e^{s_{3} u_{T}^{2}} e^{-\int_{t}^{T}\left(s_{1} u_{s}^{2}+s_{2} u_{s}\right) d s} \mid \mathcal{F}_{t}\right] .
$$

Then the Feynman-Kac stochastic representation theorem provides us that $y$ is the solution of the following PDE

$$
\frac{\partial y}{\partial t}+\tilde{\kappa}(\tilde{\theta}-u) \frac{\partial y}{\partial u}+\frac{1}{2} \tilde{\sigma}^{2} \frac{\partial^{2} y}{\partial u^{2}}-\left(s_{1} u^{2}+s_{2} u\right) y=0
$$

with a final condition

$$
y(u, T, T)=e^{s_{3} u^{2}} .
$$

Similarly $y$ can be written by

$$
y(u, t, T)=e^{\frac{1}{2} G(T-t) u^{2}+H(T-t) u+I(T-t)}
$$

and the solution of ODEs for $G(\tau), H(\tau), I(\tau)$ are given in (22), (23), and (24) in Lemma 3.3 respectively (See Schöbel and Zhu [17] for details). For $z=i \phi$, we use similar techniques to get the expression in $(21)$ for $\tilde{f}(i \phi)$, which concludes the lemma.

\section{References}

[1] G. S. Bakshi, C. Cao, and Z. W. Chen, Empirical performance of alternative option pricing models, Journal of Finance 52 (1997), no. 5, 2003-2049.

[2] D. Bates, Post-87 crash fears in the SEP 500 futures option market, Journal of Econometrics 94 (2000), 181-238.

[3] F. Black and M. Scholes, 1973, The pricing of options and corporate liabilities, Journal of Political Economy 81 (1973), 637-659.

[4] M. Chen and Y. Gau, Pricing currency options under stochastic volatility, 2004 NTU International Conference on Finance, 2004.

[5] J. C. Cox, J. E. Ingersoll, and S. A. Ross, A theory of the term structure of interest rates, Econometrica 53 (1985), no. 2, 385-407.

[6] B. Dupoyet, Information content of cross-sectional option prices: A comparison of alternative currency option pricing models on the Japanese yen, Journal of Futures Markets 26 (2006), 33-59.

[7] S. L. Heston, A closed-form solution for options with stochastic volatility with applications to bonds and currency options, The Review of Financial Studies 6 (1993), 327-343.

[8] J. Hull and A. White, The pricing of options on assets with stochastic volatilities, Journal of Finance 42 (1987), 281-300.

[9] J. Johnson and D. Shanno, Option pricing when the variance is changing, Journal of Financia1 and Quantitative Analysis 22 (1987), 143-151.

[10] I. J. Kim and S. Kim, Empirical comparison of alternative stochastic volatility option pricing models: Evidence from Korean KOSPI 200 index options market, Pacific-Basin Finance Journal 12 (2004), 117-142.

[11] Y.-N. Lin, N. Strong, and X. Xu, Pricing FTSE 100 index option under stochastic volatility, Journal of Futures Markets 21 (2001), 197-211. 
[12] A. Melino and S. Turnbull, Pricing foreign currency options with stochastic volatility, Journal of Econometrics 45 (1990), 239-265.

[13] R. C. Merton, Theory of rational option pricing, Bell Journal of Economic and Management Science 4 (1973), 141-183.

[14] S. Nandi, How important is the correlation between returns and volatility in a stochastic volatility model? Empirical evidence from pricing and hedging in the SESP 500 index options market, Journal of Banking and Finance 22 (1998), 589-610.

[15] E. Nicolato and E. Venardos, Option pricing in stochastic volatility models of the Ornstein-Uhlenbeck type, Mathematical Finance 13 (2003), 445-466.

[16] M. Rubinstein, Implied binomial trees, Journal of Finance 49 (1994), 771-818.

[17] R. Schöbel and J. Zhu, Stochastic volatility with a Ornstein-Uhlenbeck process: An extension, European Finance Review 3 (1999), 23-46.

[18] L. Scott, Option pricing when the variance changes randomly: Theory, estimation, and an application, The Journal of Financial and Quantitative Analysis 22 (1987), 419-438.

[19] _ Pricing stock options in a jump-diffusion model with stochastic volatility and interest rates: Applications of Fourier inversion methods, Math. Finance 7 (1997), 413424 .

[20] E. Stein and J. Stein, Stock price distributions with stochastic volatility: An analytic approach, Review of Financial Studies 4 (1991), 727-752.

[21] J. B. Wiggins, Option values under stochastic volatility: Theory and empirical estimates, Journal of Financial Economics 19 (1987), 351-372.

[22] J. E. Zhang and J. Shu, Pricing SEP 500 index options with Heston's model, Proceedings, 2003 IEEE International Conference on Computational Intelligence for Financial Engineering, 2003.

KYoung-SoOK MoOn

Department of Mathematics \& Information

KYUNGWON UNIVERSITY

GyeongGi-DO 461-701, KoreA

E-mail address: ksmoon@kyungwon.ac.kr

JUNG-YON SEON

Department of Mathematics

Korea UNIVERSITY

Seoul 136-701, Korea

E-mail address: sunjy24@korea.ac.kr

IN-SuK WeE

Department of Mathematics

Korea UNIVERSiTy

SEOUl 136-701, KoreA

E-mail address: iswee@korea.ac.kr

Choongseok Yoon

Department of Mathematics

Korea University

SeOUl 136-701, Korea

E-mail address: csyoon@korea.ac.kr 